\title{
Adaptive removal of power-line interference from high resolution ECG
}

\author{
Ismail Sadiq, Adil M. Zuberi, Idrees Zaman, Ammad Hassan, Tahir Zaidi
}

Centre for Advanced Research in Engineering, Islamabad, Pakistan

Email: ismail_sadiq ee@yahoo.com, amz369@hotmail.com, idreeszaman@yahoo.com, ammadhassan11@gmail.com, tahir.zaidi@case.edu.pk

Received 1 May 2012; revised 12 June 2012; accepted 6 July 2012

\begin{abstract}
The paper presents a novel approach to adaptive removal of power-line interference, taken to be at $50 \mathrm{~Hz}$, from high resolution ECG sampled at $1 \mathrm{KHz}$. ECG data consists of 12 lead data. Each of the 12 leads contains power line interference at the time of acquisition. So power line interference needs to be removed from each lead. An approach that uses the power spectral density over the range of interest is used to determine the exact frequency of the interfering component and filter coefficients for the desired notch filter are determined using an interpolation technique.
\end{abstract}

Keywords: Notch Filter; Power Spectrum; Linear Interpolation; Adaptive Noise Removal

\section{INTRODUCTION}

Power-line interference is a common source of noise encountered during ECG acquisition. It happens due to the frequency component of the $\mathrm{AC}$ mains that occurs at $50 / 60 \mathrm{~Hz}$ (depending on the region). The problem is that this frequency component does not occur exactly at 50 Hz. So a fixed notch filter of $50 \mathrm{~Hz}$ will notch effectively remove the noise component effectively even if it occurs at 49 or $51 \mathrm{~Hz}$. The paper proposes a technique which first locates the peak that occurs in the region of interest and estimates the coefficients of the filter required to remove the detected noise component. The technique is not just limited to noise detect at $50 \mathrm{~Hz}$ it can be used to detect and remove noise at any frequency adaptively. The algorithm is also efficient as it only calculated the frequency spectrum over the region of interest and hence it is not computationally intensive.

\section{METHOD}

10 second data of ECG sampled at $1 \mathrm{KHz}$ is used as an input. The DFT is evaluated over these 10,000 points for the frequency range of 45 to $55 \mathrm{~Hz}$. A fewer number of points can also be used while calculating the DFT (but at a slight expense of accuracy). The formula for the DFT (given in [1]) is given below:

$$
X(k)=\sum_{n=1}^{N} x(n) w_{N}^{(n-1)(k-1)}
$$

where $x(n)$ corresponds to the input signal of ECG data, $N$ corresponds to the total number of points $(10,000$ in our case),

$$
w_{N}=e^{\frac{-j 2 \pi}{N}}
$$

And $k$ corresponds to the component of the DFT. It ranges from the value corresponding to $45 \mathrm{~Hz}$ to the value corresponding to $55 \mathrm{~Hz}$. It can be calculated from the following formula:

$$
k=\frac{N}{F S} * f
$$

" $F S$ " is the sampling frequency, " $N$ " is the number of points In the DFT and $\mathrm{f}$ the frequency for which $k$ is to be calculated.

From the calculated DFT, the max point in the range of $45-55 \mathrm{~Hz}$ and the corresponding frequency is determined. The coefficients for a notch filter with that frequency are determined using an interpolation technique. The notch filter is a $2^{\text {nd }}$ order IIR filter with numerator and denominator polynomials going up to $2^{\text {nd }}$ order. All the coefficients are the same for notch filters in the range of 45 to $55 \mathrm{~Hz}$ except for the $1^{\text {st }}$ order, corresponding to $(n-1)$, denominator and numerator coefficient (which are identical) but vary linearly as the frequency of the notch is increased. Figure 1 shows a plot of the $1^{\text {st }}$ order coefficient as the frequency increases from 45 to $55 \mathrm{~Hz}$.

The trend followed by the coefficients is almost linear; hence linear interpolation can be used to estimate the $1^{\text {st }}$ order coefficient for a frequency that lies within this range.

The formula used for $F s=1 \mathrm{KHz}$ and a bandwidth of $1 \mathrm{~Hz}$ is given in (4): 


$$
\text { coeff } 2=(f-45) / 10 *((-1.86388191743263)-(-1.90233884591712))+(-1.90233884591712) \text {; }
$$

where $\mathrm{f}$ is the detected frequency and -1.86388191743263 and -1.90233884591712 are the first order coefficients for the notch filter at frequencies of 55 and $45 \mathrm{~Hz}$ respectively.

\section{RESULTS}

Figure 2 shows the FFT for an ECG data sample over the range of 45 to $55 \mathrm{~Hz}$. The spike exists exactly at
49.13 Hz. The adaptive algorithm we implemented estimated the spike at $49.1333 \mathrm{~Hz}$ and removes this noise, after estimating the coefficients for the notch filter, as shown in Figure 3.

\section{CONCLUSION}

A technique for removing noise at specific frequencies through notch filters has been implemented. A high

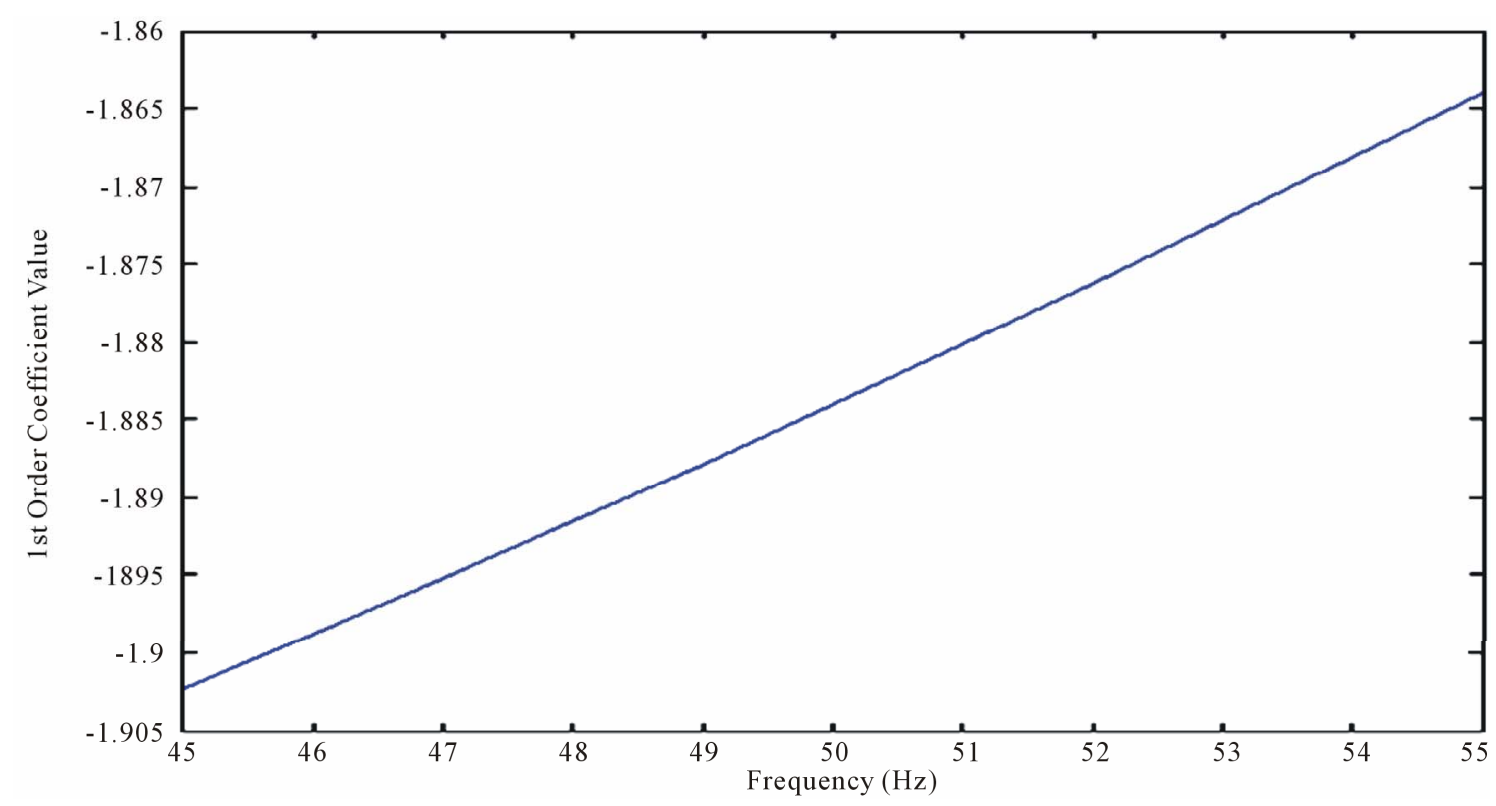

Figure 1. $1^{\text {st }}$ Order Coefficient vs Frequency plot for notch filter designed for $1 \mathrm{KHz}$ sampling frequency.

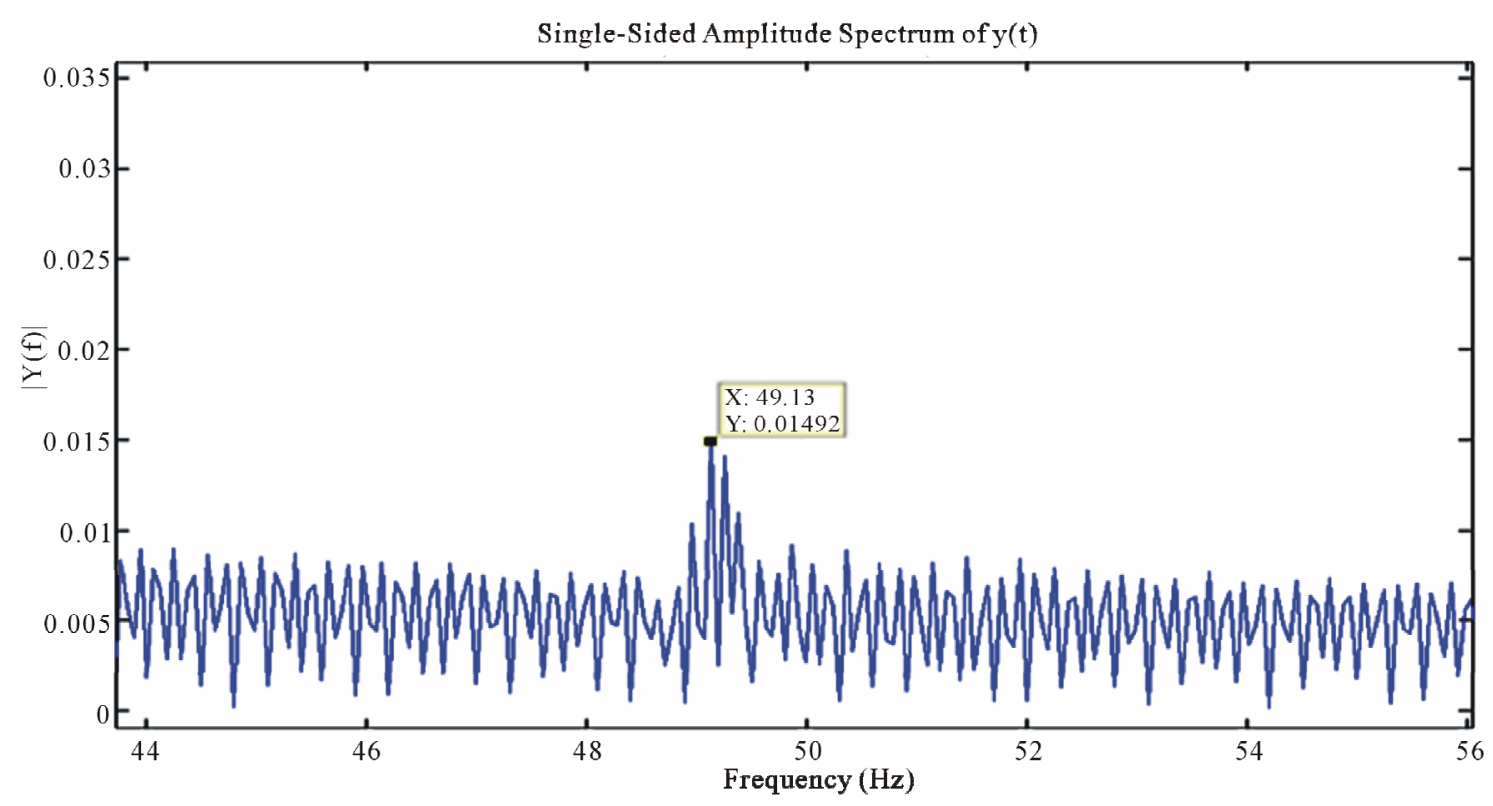

Figure 2. FFT for raw ECG Data acquired at $1 \mathrm{KHz}$ over the range of 45 to $55 \mathrm{~Hz}$ (x-axis: Frequency in $\mathrm{Hz}$, $\mathbf{y}$-axis: Power Spectrum). 


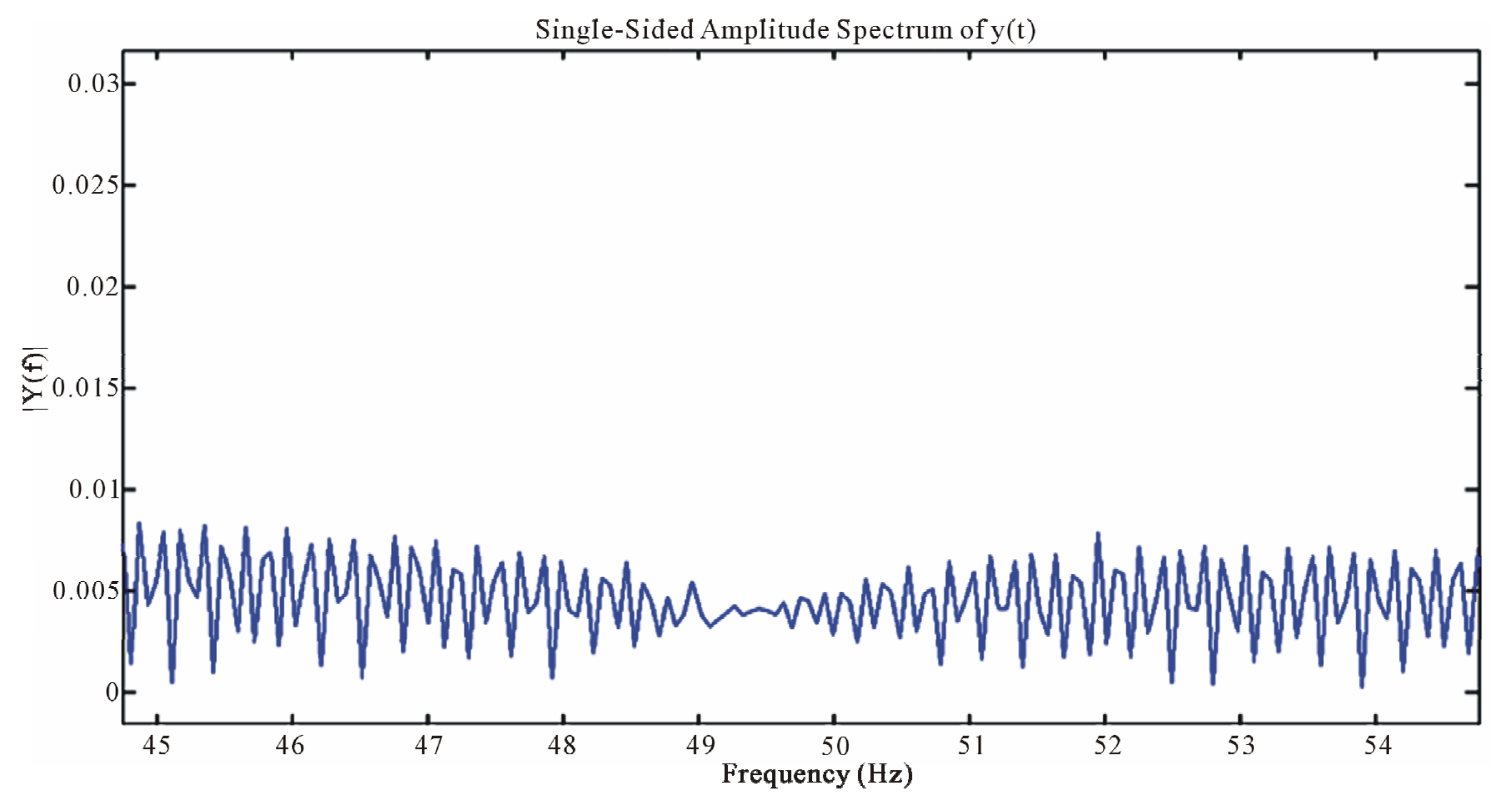

Figure 3. FFT for ECG Data acquired at $1 \mathrm{KHz}$ over the range of 45 to $55 \mathrm{~Hz}$ after noise at $49.13 \mathrm{~Hz}$ is adaptively removed (x-axis: Frequency in Hz, y-axis: Power Spectrum).

number of points while calculating the DFT leads to an accurate estimation of the frequency of the noise. The technique does not require a desired signal as is required in traditional adaptive techniques [2-4] and hence can be implemented on any sort of data.

\section{REFERENCES}

[1] Proakis, J.G. and Manolakis, D.G. (1996) Digital signal processing. 3rd Edition, Prentice Hall, Upper Saddle River, 343-345.
[2] Olguín, D.O., Bouchereau, F. and Martínez, S. (2005) Adaptive notch filter for EEG signals based on the LMS algorithm with variable step-size parameter. Conference on Information Sciences and Systems, Baltimore, 16-18 March 2005.

[3] Mihov, G. (2011) Subtraction procedure for removing powerline interference from ECG: Dynamic threshold linearity criterion for interference suppression. The 4th International Conference on Biomedical Engineering and Informatics (BMEI), Sofia, 15-17 October 2011.

[4] Malindi, P. (2002) Cancelling power line interference in electrophysiological signals. ECT Research Journal, 2. 